\title{
P I I-2 I. Induction of persistent mucosal humoral and cellular responses following immunization of mice with HIV-I envelope protein in inulin-derived adjuvants
}

\author{
AD Cristillo*1, G Ferrari ${ }^{1}$, L Hudacik ${ }^{1}$, D Thompson ${ }^{1}$, B Bowen ${ }^{1}$, B Lewis ${ }^{1}$, \\ L Galmin ${ }^{1}$, J Suschak ${ }^{1}$, N Petrovsky ${ }^{2}$, P Markham ${ }^{1}$ and R Pal ${ }^{1}$
}

\author{
Address: ${ }^{1}$ Advanced BioScience Laboratories, Inc., Kensington, USA and ${ }^{2}$ Australian National University Medical School, Canberra Hospital, \\ Woden ACT 2606, Australia \\ * Corresponding author
}

from AIDS Vaccine 2009

Paris, France. 19-22 October 2009

Published: 22 October 2009

Retrovirology 2009, 6(Suppl 3):PI66 doi:10.1 186/1742-4690-6-S3-PI66

This abstract is available from: http://www.retrovirology.com/content/6/S3/PI66

(c) 2009 Cristillo et al; licensee BioMed Central Ltd.

\section{Background}

Since HIV transmits primarily via mucosal route during sexual transmission; an ideal HIV-1 vaccine should induce both systemic and mucosal humoral and cellular responses. Although HIV envelope formulated with QS21 or Alum elicits strong systemic antibody responses, mucosal antibody and T-cell responses are generally weak. Herein we examined the systemic and mucosal immune responses elicited following a DNA prime/protein boost HIV vaccine formulated with inulin-derived (Advax) adjuvants. Such adjuvants have been shown to be less reactogenic and can induce immune responses in systemic and mucosal compartments.

\section{Methods}

BALB/c mice were primed with plasmid DNA encoding HIV Env BaL by intramuscular route (IM) at week 0, 2 and 4 and boosted with gp120 protein formulated with different Advax adjuvants administered via IM or intranasal (IN) at week 9 and 11 either alone or in combination.

\section{Results}

Immunization with gp120 formulated in Advax-M adjuvant by IN route elicited anti-Env antibodies in serum and mucosal secretions including vaginal washes, fecal pellets, lung washes and saliva and this response was significantly enhanced in DNA primed animals. Although IgA antibodies were detected in mice immunized systemically with
Advax-P adjuvant, levels were lower than that observed with Advax-M adjuvant. Use of both adjuvants where mice were immunized with gp120 formulated with Advax-M by IN route followed by Advax-P adjuvant by IM route or vise versa also elicited strong IgA responses in systemic and mucosal compartments persisting for a prolonged period. Robust induction of Th1 cytokines was noted following DNA prime/protein boost immunization as compared to protein only immunization with Advax-M or with the combination adjuvant approach.

\section{Conclusion}

These findings demonstrate that the DNA prime/protein boost vaccine strategy for HIV using inulin-based adjuvants elicits immune responses in systemic and mucosal compartments, thereby providing broad immunological responses for the protective efficacy against mucosal transmission of HIV-1. 